\title{
Observations of Correlated Behavior of Two Light Torsion Balances and a Paraconical Pendulum in Separate Locations during the Solar Eclipse of January 26th, 2009
}

\author{
A. F. Pugach ${ }^{1}$ and D. Olenici ${ }^{2}$ \\ ${ }^{1}$ Main Astronomical Observatory of National Academy of Sciences, 03680 Kiev, Ukraine \\ ${ }^{2}$ Astronomical Observatory, University of Stefan cel Mare, 720229 Suceava, Romania
}

Correspondence should be addressed to A. F. Pugach, pugach@mao.kiev.ua

Received 8 September 2011; Revised 15 November 2011; Accepted 29 November 2011

Academic Editor: J. Javaraiah

Copyright () 2012 A. F. Pugach and D. Olenici. This is an open access article distributed under the Creative Commons Attribution License, which permits unrestricted use, distribution, and reproduction in any medium, provided the original work is properly cited.

\begin{abstract}
On January 26th, 2009, simultaneous observations of the reactions of two very light torsion balances (Kiev, Ukraine) and a paraconical pendulum (Suceava, Romania, $440 \mathrm{~km}$ away) were performed during a solar eclipse that was not visible at those locations but only in the Indian Ocean. Significant correlation between the behavior of the torsion balances and the pendulum was observed. The conclusion is that a solar eclipse influences the reactions of torsion balances and pendulums, even in areas of the Earth where it is not optically visible.
\end{abstract}

\section{Introduction}

It has repeatedly been reported that during solar eclipses nonconventional physical effects are sometimes observed. During a total solar eclipse on June, 30th 1954, the Nobel laureate Maurice Allais observed an abrupt deviation of the oscillation plane of a short paraconical pendulum [1], and in 1959, he observed a similar effect on a weaker level. These reports stimulated the further search for nonconventional effects associated with solar eclipses. In order to investigate the anomalies arising during a syzygy, a number of experiments have been performed that appeared to show variation of the gravitational constant $[2,3]$, and one experiment appeared to show change of the atomic frequency standards during a solar eclipse in December 1992 [4].

However the most easily repeated experiments are relatively simple ones with torsion balances of different designs and forms including horizontal and vertical, and experiments with a short pendulum supported on a ball (paraconical) and with tilt-meters and gyroscopes [5-7]. Several dozens of such experiments appear to confirm the reality of various different anomalies but have not provided any clear answer to the question of how frequent and unique anomalies really are. Despite the numerous positive conclusions, in some cases, ambiguous and even negative results have been obtained [8]. The complexity of the issue is exacerbated by the fact that the circumstances of solar and lunar eclipses never repeat, so that each eclipse evolves under unique conditions different from those of all previous eclipses. Besides, no high (certified) standard exists for the measuring apparatus, and current equipment is mainly manufactured in a semiartisanal manner.

Nevertheless, Duif has attempted to analyze the available pool of provisional findings and has arrived at the overall conclusion that the most likely common factor behind all the successes and failures is a real phenomenon of some influence of solar eclipses on the reaction of physical devices that cannot be adequately explained in the current framework of Newtonian and relativistic physics [9].

In this publication, we present the results of synchronized measurements with very light torsion balances and with a paraconical pendulum that we obtained during the solar eclipse of January 26th, 2009. 


\section{Description of the Instruments and the Measurement Methods}

2.1. Torsion Balance. In our experiments, we use miniature torsion balances (TBs), the mass of whose mobile element does not exceed $0.5 \mathrm{~g}$. A straw of $110 \mathrm{~mm}$ length serves as an asymmetrical beam, at the end of whose short arm (1) a lead counterweight of mass $M$ is attached. This mobile beam is suspended on a thin monofilament of natural silk $(d \approx 20 \mu)$. A visible pointer is attached to the long $\operatorname{arm} L$ (mass $m$ ).

For this device, the following relation holds:

$$
M^{*} l=m^{*} \mathbf{L}
$$

A more-detailed description of the instrument and its characteristics can be found in [10].

The mobile part of the torsion balance (beam, pointer, and counterweight) is suspended inside a sealed glass housing. An external webcam registers movement of the beam. Images sent by the webcam to a computer are analyzed by a special program which, once every minute, records the azimuth of the beam and the time (UT). This registration system has good noise immunity and provides high accuracy readings with the average error of one measurement being around $\pm 0.175^{\circ}$, that is, about 10 arc minutes [11].

In addition, direct observations or statistical analysis of the observations revealed that TB does not respond to changes in

(i) gravitation potential;

(ii) temperature of the device itself;

(iii) meteorological parameters (wind, pressure, humidity);

(iv) static electric field;

(v) moderate magnetic field;

(vi) degree of ionization of the ionosphere;

(vii) operation of electromechanical units;

(viii) change in the load on the TB thread;

(ix) possible microvibration of the floor;

(x) sound waves of moderate intensity;

(xi) radio waves and cell phones;

(xii) convective air movement inside the TB housing to a minimum; and it may not be the cause of significant vibration readings.

Five days before the eclipse, two nearly identical apparatuses WEB-1 and WEB-2 were installed in an insulated, heated, and dry room with tightly closed windows and doors. Access to the room was only available to the leader of the experiment (the first author).

Measurements began on 23 January at $17 \mathrm{~h} \mathrm{UT}$ and lasted about 6 days without interruption, up to $23 \mathrm{~h} 59 \mathrm{~m}$ UT on January 28. Thus, the reaction of the TBs to the surrounding circumstances was tracked for about 3 days before the eclipse and 2.5 days after. The eclipse on Earth began on 26 January at $4 \mathrm{~h} 56 \mathrm{~m} \mathrm{UT}$ and ended at $11 \mathrm{~h} 00 \mathrm{~m} \mathrm{UT}$. The geocentric

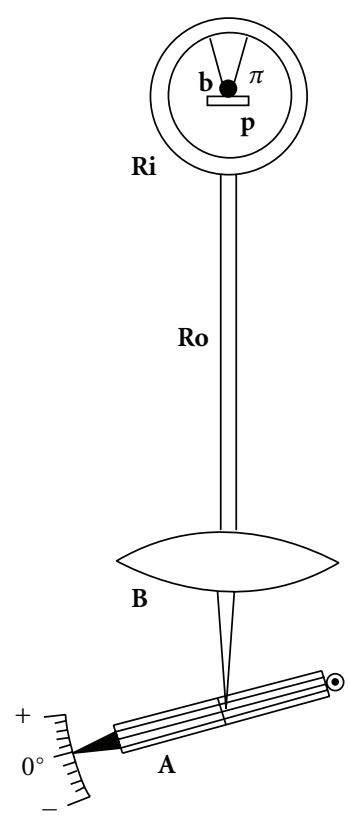

Figure 1: A schematic representation of the PP experiment.

conjunction of the Sun and the Moon in right ascension took place at $8 \mathrm{~h} 06 \mathrm{~m}$ UT. Since the eclipse was neither visible in Ukraine nor in Romania, no times of local maximum phase were defined.

2.2. Paraconical Pendulum. As well as using conventional Foucault pendulums, recently, several observers of syzygy effects have begun to use shorter pendulums equipped with a special suspension proposed by Maurice Allais, in which the pendulum is supported upon a small highly accurate steel ball. Such a pendulum not only can oscillate in two perpendicular planes but also can rotate around the vertical axis.

Our paraconical pendulum consists of a $13.3 \mathrm{~kg}$ bob $\mathbf{B}$ made from brass in a lenticular form $24 \mathrm{~cm}$ in diameter and $6 \mathrm{~cm}$ in thickness, a suspension rod Ro also of brass (68 cm length, $3.3 \mathrm{~cm}$ diameter and $5 \mathrm{~kg}$ mass), and an upper suspension ring $\mathbf{R i}$ of aluminium (inner diameter $15 \mathrm{~cm}$, outer diameter $24 \mathrm{~cm}, 5 \mathrm{~cm}$ thick, and $3.7 \mathrm{~kg}$ mass). A pivot $\pi$ is fixed at the inner side of the upper part of the ring Ri. This pivot rests upon a highly accurate steel ball $\mathbf{b}$ (diameter $6 \mathrm{~mm}$ ) which rolls upon a highly planar support plate $p$. The total mass of the pendulum is $22 \mathrm{~kg}$. The distance between the point of oscillation and the centre of the bob is $85 \mathrm{~cm}$. The motion is monitored by an alidade $\mathbf{A}$ that reads to an accuracy of $12^{\prime}$ of arc. One of these pendulums (Figure 1) has been permanently installed in the Planetarium of Stefan cel Mare University, Suceava, Romania.

Also, an automated identical paraconical pendulum was installed in another place in the Planetarium.

The results obtained with these two pendulums, one monitored manually and the other automatically, were identical. This fact demonstrates that the human factor does not influence the behavior of the pendulum. An example is 


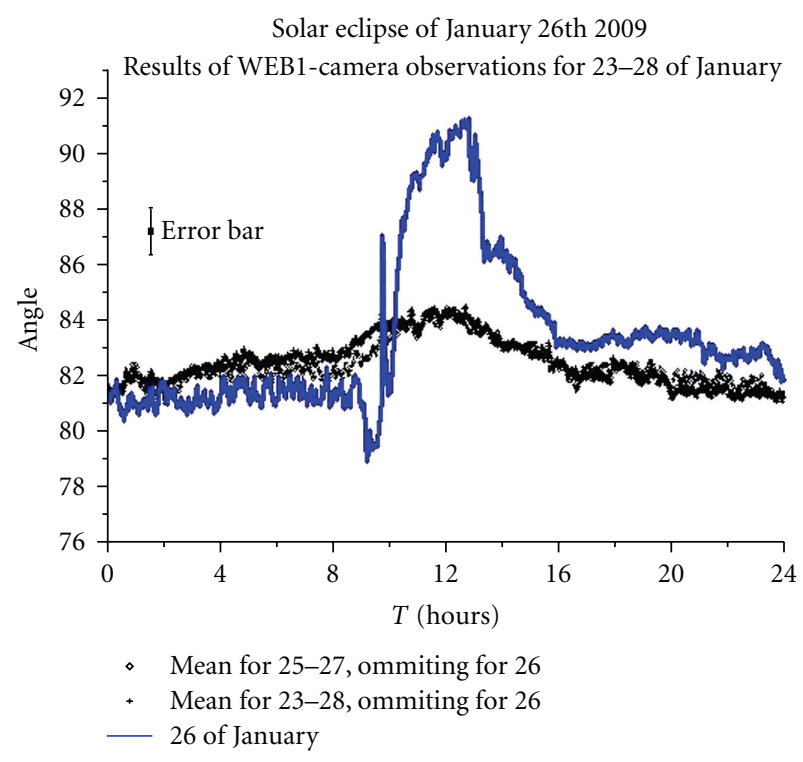

FIGURE 2: WEB-1 observations: the averaged daily variation (in black and grey) and the variation on January 26th, 2009, the day of the solar eclipse (in blue). $T$ is local (Kiev) time.

the graphs obtained with these two pendulums during the solar eclipse of August 1st, 2008 [12].

\section{Measurements}

3.1. Torsion Balances (TBs). The results obtained on 23, 24, 25,27 , and 28 January are considered as being background or baseline measurements. They allow us to determine the response of the devices during exterior circumstances free of any eclipse effect. The analysis showed that in these days nothing particularly significant was registered, except for the usual minor daily variations.

Figure 2 shows the average background WEB-1 observations for January 25, and 27 (narrow window background, black) and the average background for January 23, 24, 25, 27 , and 28 (wide window background, grey). A comparison of these wide- and narrow-window background curves shows that they are almost completely similar, so it is arguable that the background varied in the same manner on January 26the eclipse day - and that the mean background value of these five days can be applied to the day of the eclipse. The continuous blue line in this figure shows the measurements on the eclipse day, that is, January 26.

The reduced "clean" result after subtracting the mean background is shown in Figure 3. The vertical lines on the graph indicate the main stages of the eclipse.

A similar result was obtained by observations with the instrument WEB-2 over the period 23-28 January. The procedure described above for measuring and subtracting the background fluctuations provided a clean result when applied to the WEB-2 observations, thus eliminating the influence of daily variations. Comparison of the "pure" results obtained with the instruments WEB-1 and WEB-2 is shown in Figure 4. These results are qualitatively similar and

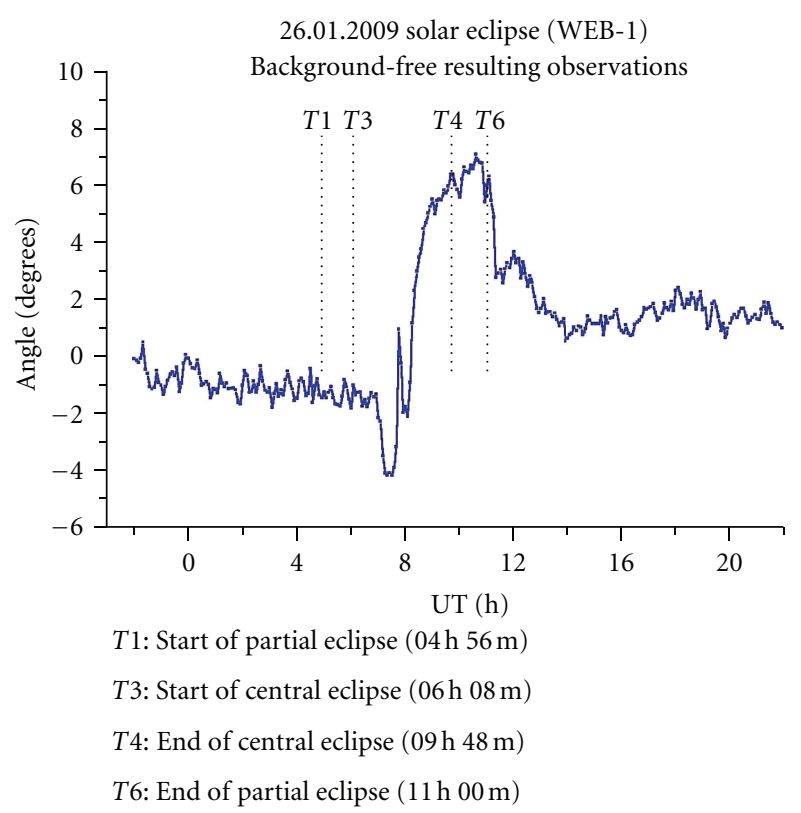

Figure 3: Record of the solar eclipse freed from the influence of normal daily variations.

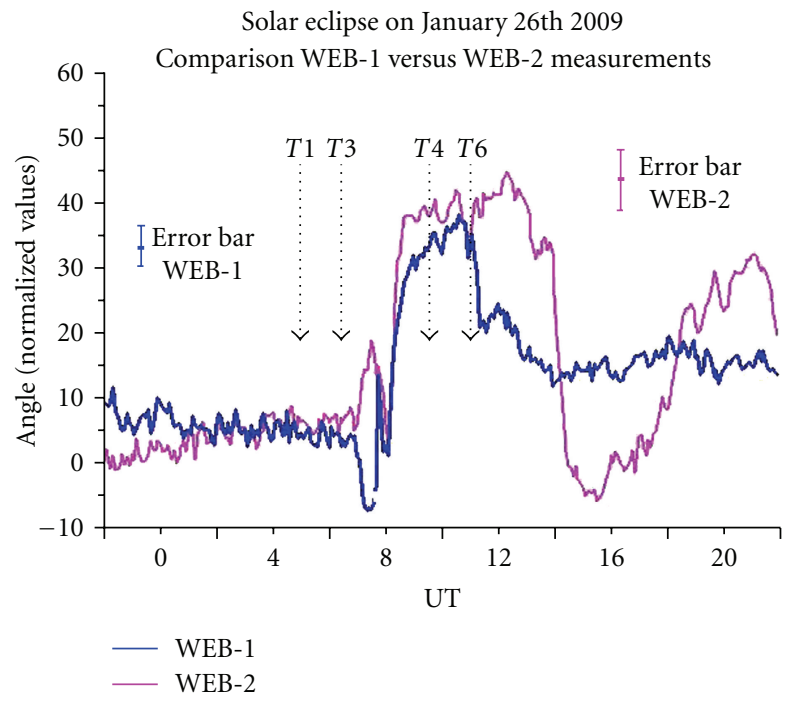

FIgURE 4: Comparison of the "pure" results obtained with WEB-1 and WEB-2 devices on 26.01.2009.

obviously have a high correlation coefficient within time interval $T 1-T 6$.

3.2. Paraconical Pendulum (PP). Figure 5 shows the changes of the PP oscillation plane in the period from $22 \mathrm{~h} 00 \mathrm{~m}$ UT (25.01) to $14 \mathrm{~h} 24 \mathrm{~m}$ UT on 26 January. The azimuth is measured from south, but this is not particularly important, because these measurements as well as the TB measurements are essentially qualitative in nature, and therefore the angular reference system can be chosen arbitrarily.

In Figure 5, the numbers from 1 to 85 are the serial numbers of consecutive measurements of the change of the oscillation plane azimuth of the pendulum over 55 minutes. 


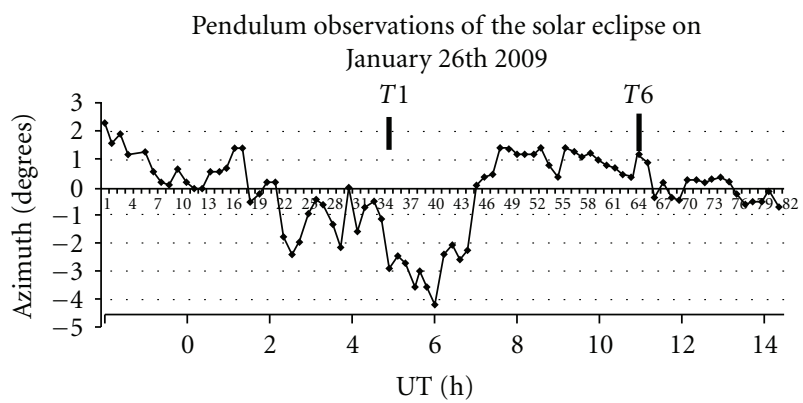

Figure 5: Changes in the pendulum oscillation plane on the date of the solar eclipse. Numbers on the linear scale in the center of the figure indicate the sequential number of measurements that were taken every 11 minutes.

The determinations began on 25.01 at $21 \mathrm{~h}$ UT and ended on 26.01 at $15 \mathrm{~h} 55 \mathrm{~m} \mathrm{UT}$.

It is well known that over time, due to the force of friction, the amplitude of oscillation is damped, and also the oscillations become very elliptical.

In order to eliminate these problems, the pendulum was started once every hour, during which the damping of the amplitude of the oscillations was small and the oscillations remained almost linear. In every hour, five readings were taken at intervals of 11 minutes, and the last five minutes was used for preparing to restart the pendulum.

The most negative values were observed in the period between measurement number 35 (04 h $48 \mathrm{~m} \mathrm{UT}$ ) and measurement number 45 ( $06 \mathrm{~h} 48 \mathrm{~m} \mathrm{UT})$. The extreme value of $-4.2^{\circ}$ was seen at $06 \mathrm{~h} 00 \mathrm{~m} \mathrm{UT}$, that is, more than $100 \mathrm{mi}-$ nutes before the geocentric Sun/Moon conjunction.

The pendulum was operated in a chamber at Suceava Planetarium, where the temperature was substantially constant at $20^{\circ}$. Also outside (as can be checked via the Wolfram Alpha service), the temperature on January 26th, 2009 varied very little (as is normal during winter at our location). The air pressure was not constant on that day but increased a few millibars quite steadily with no relation to the eclipse (which was not visible in Suceava).

\section{Analysis of Results}

Since all the measurements were essentially qualitative in nature and no theoretical or quantitative model of the phenomenon yet exists, the only possible procedure for analysis is comparative. A positive outcome of the analysis would be to demonstrate that the rotations of the $\mathrm{TB}$ arms and the deflection of the plane of oscillation of the pendulum during this solar eclipse were not aleatory, but resulted from some external cause, the nature and structure of which remain to be elucidated.

Figure 4 shows that in the interval $T 1-T 6$, the readings of WEB- 1 and WEB-2 varied in a similar manner. Figure 6 shows how these readings of WEB-1 and WEB-2 correlate. Calculations show that, in this time interval, the correlation coefficient CC $=0.892$. Such a high value of CC indicates that the reactions of the WEB- 1 and WEB-2 devices were not

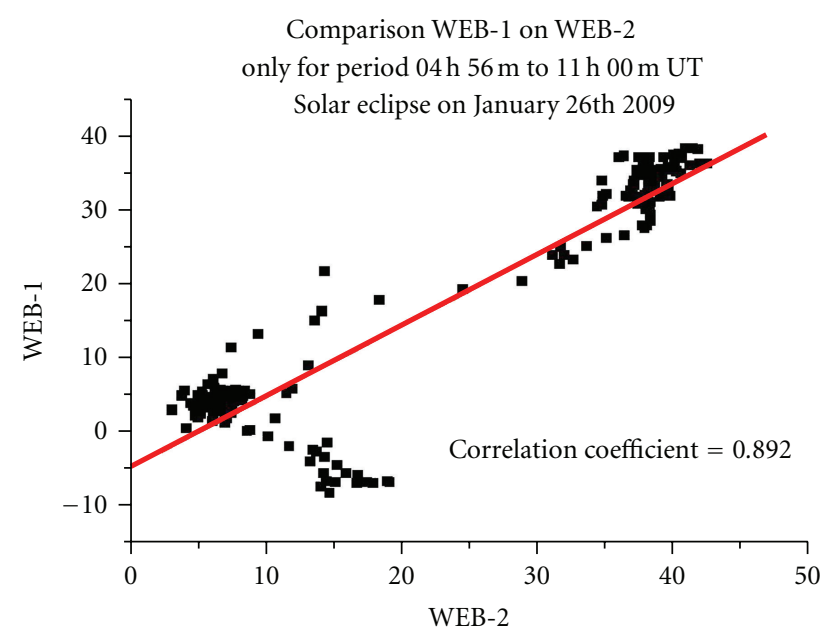

FIgure 6: Correlation of readings of WEB-1 versus WEB-2 during the time interval T1-T6.

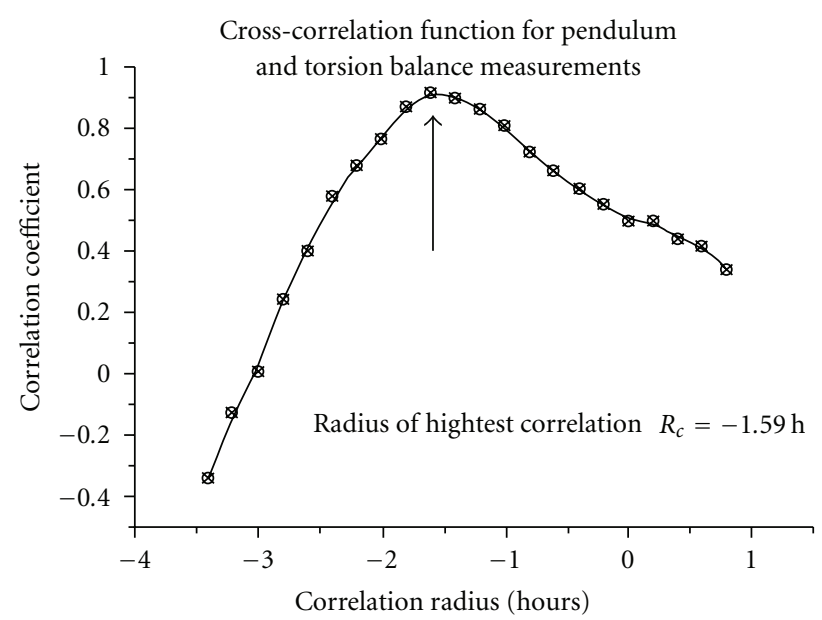

FIGURE 7: Cross-correlation function for the series of pendulum and torsion observation.

random. This conclusion is confirmed by the fact that such correlation is absent outside of the time interval $T 1-T 6$. It may appear that in the time interval $6.9 \mathrm{~h}-7.8 \mathrm{~h} \mathrm{UT}$, there was no correlation between WEB-1 and WEB-2, but, in fact, this is not the case. The reactions of the devices remained strictly correlated, but the sign of the correlation was reversed.

Comparison of the PP and the TB observations is of particular interest.

From preliminary visual comparison of the results, it appeared that the TB graph in the interval T1-T6 almost exactly repeated the PP graph, the latter being somewhat earlier. In order to clarify the value of this temporal advance, an crosscorrelation analysis of the two series of measurements (TB and PP) was carried out. The results of the calculations are shown in Figure 7.

The crosscorrelation function reaches a maximum at a correlation spacing close to one and a half hours. An accurate numerical analysis shows that the correlation spacing is 1.59 hours, the correlation coefficient being equal to 0.921 . This 


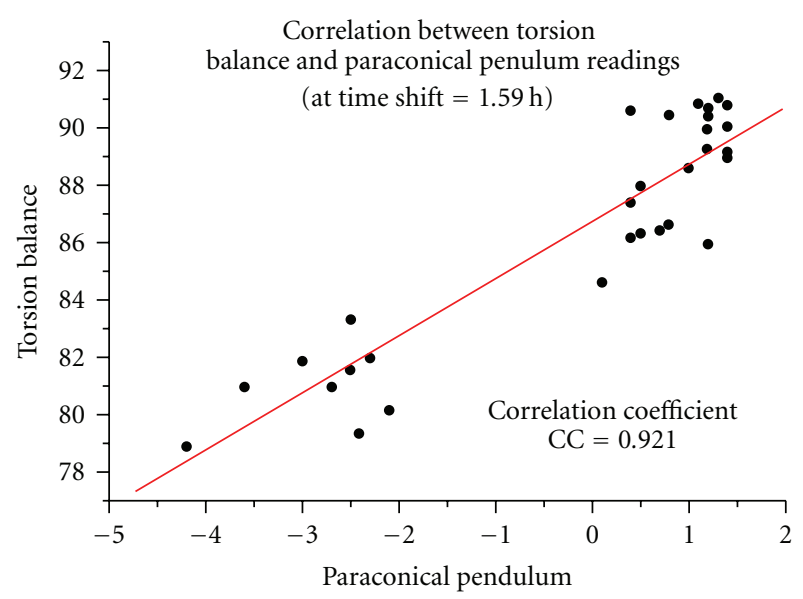

Figure 8: Correlation and the correlation coefficient between these series of observations at a correlation spacing of 1.59 hours.

means that the observations with the TB repeated the observations with the PP with a very high degree of similarity, but lagging behind in time by 1 hour 35.4 minutes.

In order to confirm the high degree of similarity of the results of the $\mathrm{PP}$ and $\mathrm{TB}$ observations in the range T1-T6, the correlation coefficient CC between these two curves was calculated. It was found to be CC $=0.921$ (see Figure 8), suggesting that these two curves are strongly mutually dependent.

The above data gives grounds to assert that the changes in the azimuth of the paraconical pendulum oscillation plane and the changes in the angles of the TB beams on January 26th, 2009 were not accidental. The behavior of the two TBs indicates that, in the period from $-2 \mathrm{~h}$ to $11 \mathrm{~h}$ UT, the beam angle variations were similar in nature. Moreover, in the time interval $T 1-T 6$, the TB pointer variations coincided with the variations of the PP oscillation plane. Such coincidence of the results of different instruments clearly indicates the presence of some dominating signal and excludes any assumption of accident.

To illustrate the high degree of correlation of the results between these essentially different types of apparatus (the TBs and the pendulum), we present Figure 9, which compares the results of measurements with instruments WEB-1 and WEB-2 (blue and purple) with the results of the pendulum measurements in the interval T1-T6 (red line) shifted by 1.59 hours. This artificial time shift at 1.59 an hour is introduced to demonstrate how similar the graphs are, taking into account that the observations were carried out with different instruments in different places. Apart from the overall character of the graphs, some smaller details are also identical as, for example, peaks at approximately $7.8 \mathrm{~h} \mathrm{UT}$.

However, the most important point is that the very high correlation between WEB-1, WEB-2, and the paraconical pendulum was only noted in the interval $T 1-T 6$, that is, from the beginning of the solar eclipse on the Earth up to its termination. Outside this interval, the correlation disappeared. From this fact, it can unequivocally be concluded that the solar eclipse was a determining factor for the readings.

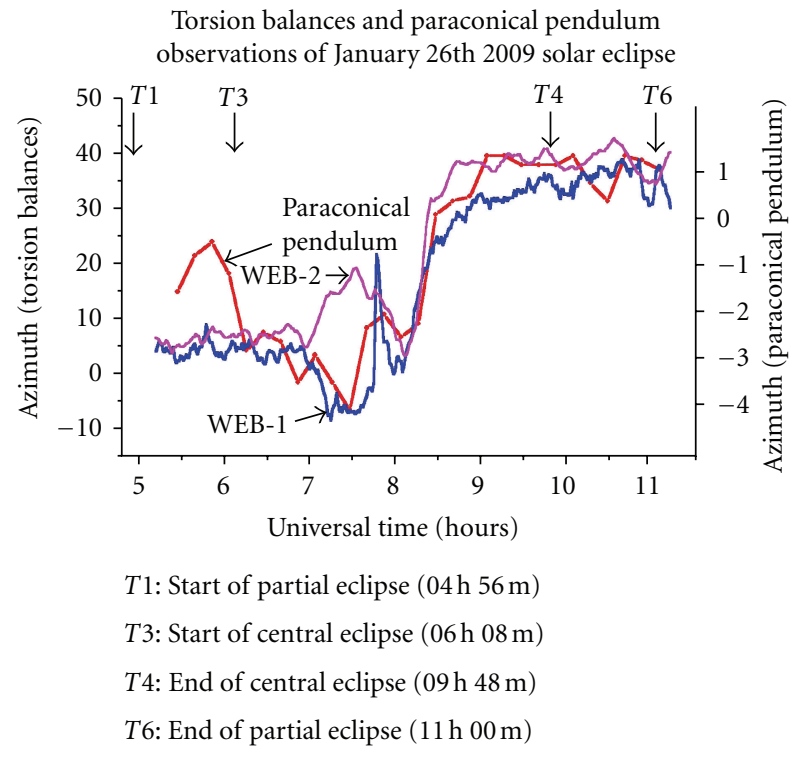

FIGURE 9: Comparison of the results of observations in Kiev (blue and purple) with observations in Suceava (red line). In order to align the curves, the graph for the PP is shifted forward by $1.59 \mathrm{~h}$.

These nonconventional solar eclipse observations have shown that this phenomenon is accompanied by effects that cannot yet be explained within the current physical picture of the world.

Comparing these observations with other available data collected from previous years reveals several features that characterize, to some extent, all the observations.

(1) Within a few hours before the solar eclipse, the readings of devices are minimized and remain stable within a narrow fluctuation band. This trend is clearly seen in observations of some past solar eclipses [13].

(2) The most significant fluctuations of the arm pointer do not coincide, as a rule, with the optical phenomenon maximum.

(3) In most cases, an active reaction of the TB to an eclipse seems first to be manifested as a pointer rotation clockwise as seen from above, with subsequent relaxation in the opposite direction afterwards.

(4) In most cases, when several instruments were used, their reactions were similar to each other, but always there were significant differences of unknown nature. We consider that, most likely, this is associated with differences of the devices from one other.

\section{Conclusion}

Another independent confirmation has been obtained of the previously established fact that at the time of solar eclipses, a specific reaction of the torsion balance can be observed. During a solar eclipse, the readings of two neighboring TBs seem to be correlated. This fact demonstrates the nonaleatory 
character of the reactions of TBs. Consequently, the reaction of these devices is deterministic, not random. A solar eclipse is such a determinant, since upon termination of a solar eclipse, the correlation becomes insignificant. This conclusion is supported by the PP observations. The PP graph and the TB graphs showed obvious similarity, with the coefficient of correlation of these two independent curves being close to 1 .

This is the second collective observation, which has registered both TB reaction and $\mathrm{PP}$ reaction during an astronomical phenomenon. The first observation referred to the solar eclipse on August 1st, 2008 when simultaneous reactions of several TBs, two PPs, and one Foucault pendulum was detected [12].

It is noteworthy that a significant correlation between the reactions of the pendulum and the TBs was valid only in the time interval when the lunar shadow was passing over the Earth. Outside this time interval T1-T4, the correlation was absent. This fact is another strong argument in favor of the conclusion that the solar eclipse was the cause of the correlated variations.

The reason for the time shift between the TB and PP readings remains unclear, although it can be assumed that the shift is due to the difference in geographic coordinates of the observations. It is possible that some unknown effect in the form of a wave was moving in space at a relatively low speed (on the astronomical scale).

The effects described above are specific in themselves. Nevertheless, they cannot be contradictory to the universal laws that are actually manifested in the external universe. They organically complement a whole set of nonconventional phenomena including other phenomena observed during solar eclipses. Most of these are not yet understandable. In particular, we wonder how any physical momentum can be transferred to our instrument during a solar eclipse. Gravity can hardly suffice as an explanation even for understanding the results of the PP measurements. The gravitational potential grows slowly and smoothly over a number of days before eclipse and then declines smoothly afterwards without any sudden variations, but we see relatively short-term events. Moreover, gravity is certainly not applicable to the explanation of the results of the TB observations, since the TB is not sensitive to changes in gravitational potential.

The cause of the time lag between the response of the device in Suceava and the reactions of the devices in Kiev also remains unknown. What can be this force which acts so selectively in space and time?

The anomalies found, that defy understanding in terms of modern physics, are in line with other anomalies, described in a recently published compendium "Should the Laws of Gravitation be reconsidered?" [14]. Together, these phenomena presented suggest that the classical theory of gravity is in need of significant additions and amendments.

\section{Acknowledgments}

Both authors thank Thomas Goodey for help with the preparation of this article. A. F. Pugach thanks D. P. Vorobiov for maintenance of the apparatus, and D. Olenici thanks
Professor Stefan Pintilie and students Alina Puha and Ionel Popescu for help with the observations.

\section{References}

[1] M. F. C. Allais, "Mouvement du pendule paraconique et eclipse totale de Soleli du 30 juin 1954 ," Comptes Rendus de l'Académie des Sciences, vol. 245, pp. 2001-2003, 1957.

[2] Q. S. Wang, X. S. Yang, C. Z. Wu, H. G. Guo, H. C. Liu, and C. C. Hua, "Precise measurement of gravity variations during a total solar eclipse," Physical Review D, vol. 62, no. 4, Article ID 041101, pp. 1-3, 2000.

[3] X. S. Yang and Q. S. Wang, "Gravity anomaly during the Mohe total solar eclipse and new constraint on gravitational shielding parameter," Astrophysics and Space Science, vol. 282, no. 1, pp. 245-253, 2002.

[4] S. W. Zhou and B. J. Huang, "Abnormalities of the time comparisons of atomic clocks during the solar eclipses," Il Nuovo Cimento C, vol. 15, no. 2, pp. 133-137, 1992.

[5] T. Kuusela, "Effect of the solar eclipse on the period of a torsion pendulum," Physical Review D, vol. 43, no. 6, pp. 20412043, 1991.

[6] T. Kuusela, "New measurements with a torsion pendulum during the solar eclipse," General Relativity and Gravitation, vol. 24, no. 5, pp. 543-550, 1992.

[7] E. J. Saxl and M. Allen, "1970 Solar eclipse as "seen" by a torsion pendulum," Physical Review D, vol. 3, no. 4, pp. 823$825,1971$.

[8] L. Jun, L. Jianguo, Z. Xuerong, V. Liakhovets, M. Lomonosov, and A. Ragyn, "Observation of 1990 solar eclipse by a torsion pendulum," Physical Review D, vol. 44, no. 8, pp. 2611-2613, 1991.

[9] C. Duif, "A review of conventional explanations of anomalous observations during solar eclipses," in Proceedings of the Conference on the Pioneer Anomaly, Bremen, Germany, May 2004, Preprint gr qc/0408023.

[10] A. F. Pugach, M. M. Medvedskii, and N. N. Peretyatko, "The first experience of solar eclipse observations with a miniature torsion balance ," Kinematics and Physics of Celestial Bodies, vol. 24, no. 5, pp. 253-258, 2008.

[11] A. F. Pugach and D. P. Vorobyev, "A device for recording positions of a beam of an extralight torsion balance," Kinematics and Physics of Celestial Bodies, vol. 26, no. 6, pp. 326-330, 2010.

[12] T. J. Goodey, A. F. Pugach, and D. Olenici, "Correlated anomalous effects observed during the August 1st 2008 solar eclipse ," Journal of Advanced Research in Physics, vol. 1, no. 2, Article ID 021007, 2010.

[13] A. F. Pugach, "Observations of the astronomical phenomena by torsion balance," Physics of Consciousness and Life, Cosmology and Astrophysics, vol. 9, no. 2, pp. 30-51, 2009.

[14] H. Munera, Ed., Should the Laws of Gravitation be reconsidered? Apeiron, Monreal, Spain, 2011. 

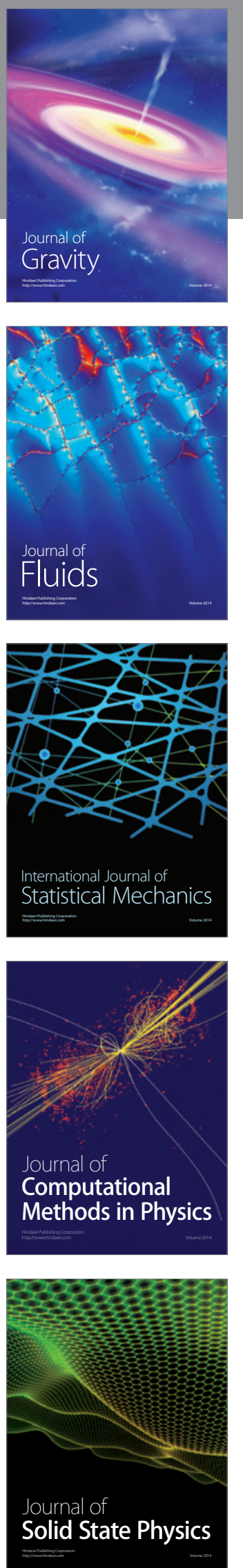

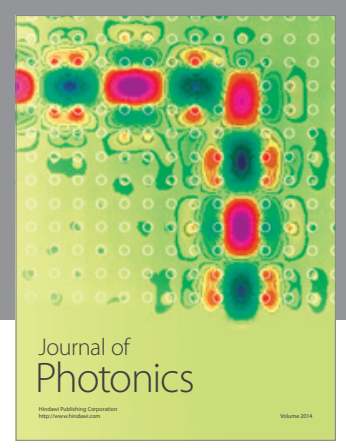

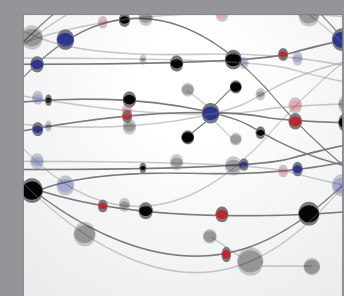

The Scientific World Journal
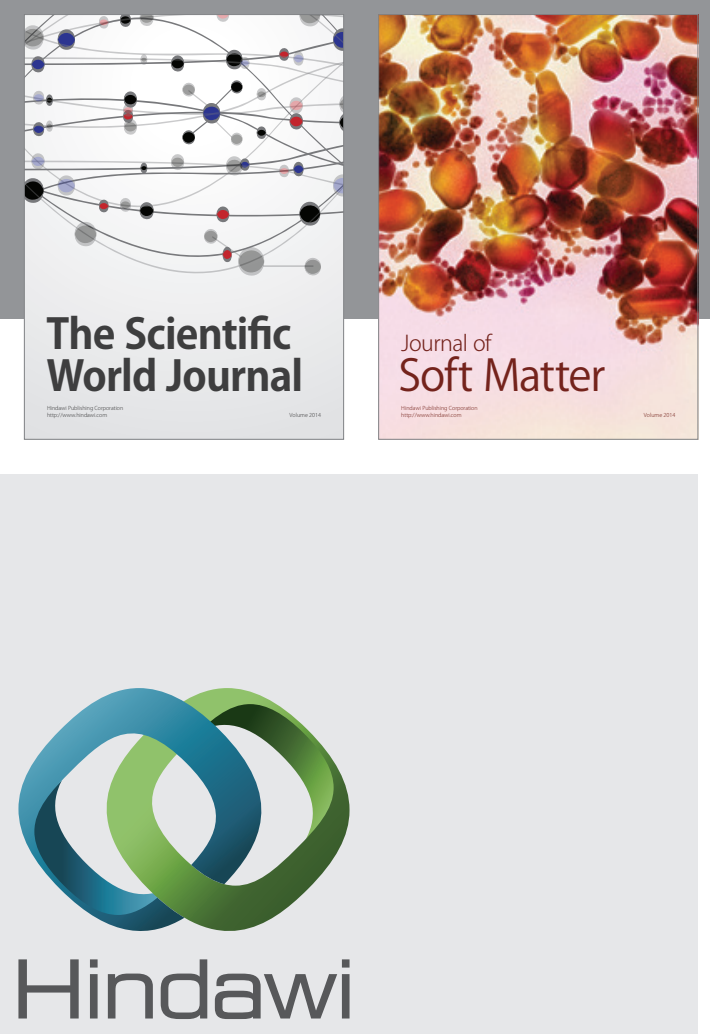

Submit your manuscripts at

http://www.hindawi.com
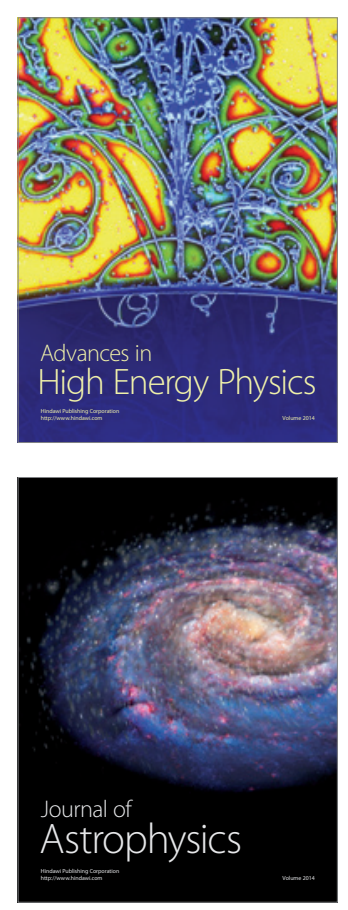
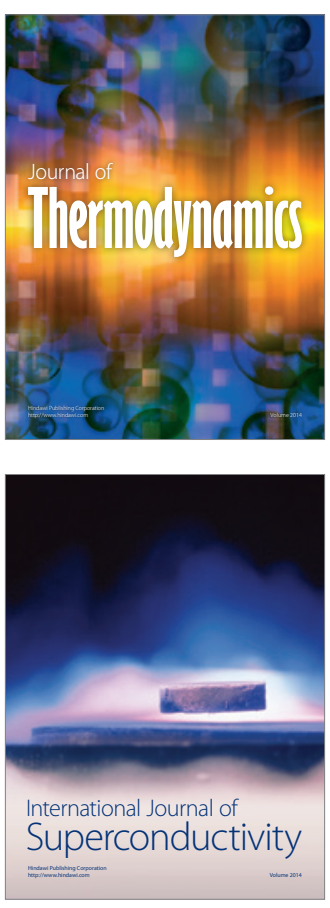
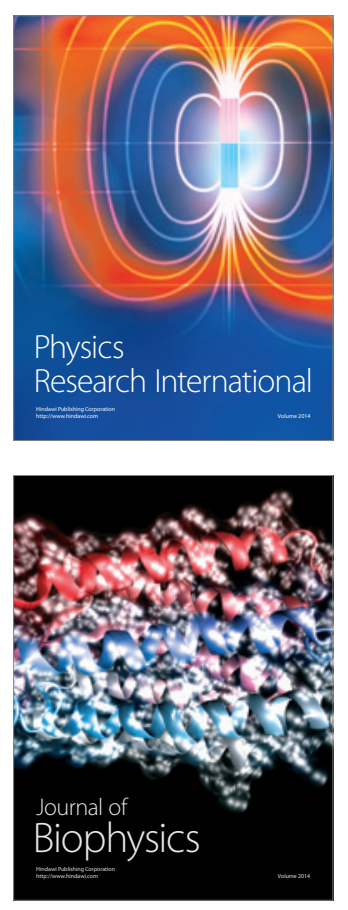
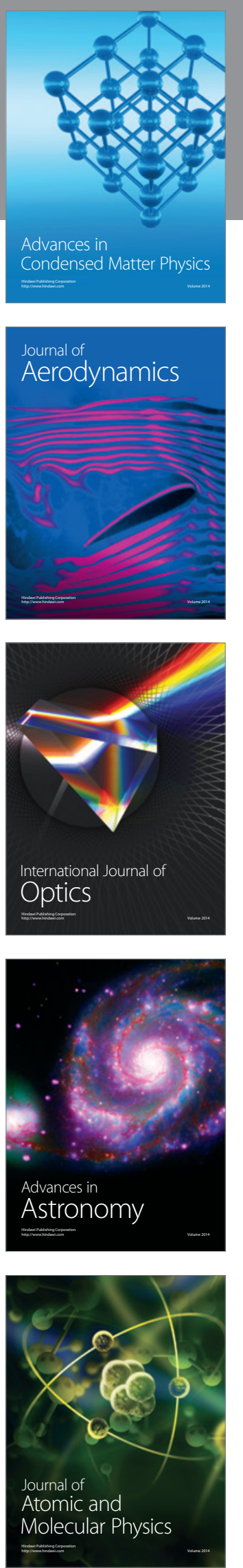